\title{
Measurements of Higgs-boson decays to leptons with the ATLAS detector
}

\author{
Lara Katharina Schildgen ${ }^{1 \star}$ on behalf of the ATLAS Collaboration
}

1 Physikalisches Institut, Universität Bonn, Nussallee 12, D-53115 Bonn

$\star$ lara.katharina.schildgen@cern.ch

Proceedings for the 15th International Workshop on Tau Lepton Physics, Amsterdam, The Netherlands, 24-28 September 2018

doi:10.21468/SciPostPhysProc. 1

\begin{abstract}
Since its discovery in 2012, the Higgs boson has served as an important probe for precision measurements of the Standard Model and for searches for new physics beyond the Standard Model. One major goal of the LHC is the precise measurement of the Higgs Yukawa couplings to fermions. The latest ATLAS results of measurements of the Higgs boson decaying to leptons are presented, namely the cross-section measurement of the Higgs boson decay to two tau leptons and the searches for the di-muon decay and leptonflavour-violating decays of the Higgs boson.
\end{abstract}

(a) (1) Copyright L. K. Schildgen et al. This work is licensed under the Creative Commons Attribution 4.0 International License. Published by the SciPost Foundation.
Received 15-11-2018

Accepted 17-01-2019

Published 19-02-2019

doi:10.21468/SciPostPhysProc.1.019

\section{Introduction}

In 2012, the ATLAS [1] and CMS [2] collaborations discovered a boson which is consistent with the Standard Model Higgs boson [3,4]. Since the discovery at the Large Hadron Collider (LHC), the properties of the Higgs boson are studied. Precise measurements of the Higgs Yukawa couplings to fermions serve as a powerful probe of the Standard Model. Here, three ATLAS analyses are presented which measure the properties of Higgs boson decays to leptons. The focus of this article will be laid on the measurement of the cross section of the Higgs boson decay to two tau leptons.

So far, the Higgs boson decay to a di-tau pair has been the only accessible leptonic Higgs boson decay mode. Evidence for the decay of the Higgs boson into two tau leptons has been found by the ATLAS and the CMS collaboration in data collected in proton-proton collisions at a centre-of-mass energy of $\sqrt{s}=7 \mathrm{TeV}$ and $8 \mathrm{TeV}$ in 2011 and 2012 [5,6]. Both collaborations observed the Higgs boson in the di-tau decay in data taken at a centre-of-mass energy of $\sqrt{s}=13 \mathrm{TeV}[7,8]$. In the following, the cross-section measurements of Higgs boson decays to pairs of tau leptons in $36.1 \mathrm{fb}^{-1}$ of proton-proton collision data taken by the ATLAS detector at a centre-of-mass energy of $\sqrt{s}=13 \mathrm{TeV}$ are presented.

The rare decay of a Higgs boson to a di-muon pair offers a good possibility to measure the Higgs coupling to second-generation fermions at the LHC. Any deviation from the SM 
prediction could be a sign of new physics. In a previous search, the ATLAS collaboration found no significant signal excess and set an observed (expected) upper limit of 2.8 (2.9) at 95\% CL on the Standard Model signal strength [9]. The CMS collaboration performed a search for the Higgs boson decaying to two muons at a centre-of-mass energy of $\sqrt{s}=13 \mathrm{TeV}$ and found the data to be compatible with the predicted background [10]. Here, an updated analysis performed by the ATLAS collaboration on the search of the Higgs boson decay to a di-muon pair is presented [11]. It is performed on data collected at $\sqrt{s}=13 \mathrm{TeV}$ which corresponds to an integrated luminosity of $79.8 \mathrm{fb}^{-1}$.

One main aim of the ATLAS experiment is to search for physics beyond the Standard Model. A signature for new physics could be lepton-flavour-violating Higgs boson decays where the Higgs boson decays into two leptons of different flavour. A previous ATLAS analysis found an upper limit of the branching ratio of the Higgs boson decaying to a muon and a hadronically decaying tau lepton to be $\mathcal{B}(H \rightarrow \mu \tau)=1.85 \%$ at 95\% CL [12]. The direct search for leptonflavour-violating Higgs boson decays [13] presented here places significantly more stringent constraints on the branching ratios than the earlier estimates. The analysis uses data taken at a centre-of-mass energy of $\sqrt{s}=8 \mathrm{TeV}$ with an integrated luminosity of $20.3 \mathrm{fb}^{-1}$.

\section{Cross-section measurements of the $H \rightarrow \tau \tau$ decay}

The preliminary analysis [7] has been performed on a proton-proton collision dataset collected in 2015 and 2016 at a centre-of-mass energy of $\sqrt{s}=13 \mathrm{TeV}$. The collected data corresponds to an integrated luminosity of $36.1 \mathrm{fb}^{-1}$. A cut-based event selection with a likelihood fit to the di-tau mass $m_{\tau \tau}$ is used.

The analysis exploits all tau decay modes (leptonic and hadronic) which leads to the channel naming scheme ' $\tau_{\text {lep }} \tau_{\text {lep }}$, ' $\tau_{\text {lep }} \tau_{\text {had }}$ ' and ' $\tau_{\text {had }} \tau_{\text {had }}$ ' for the full-leptonic, the semi-leptonic and the full-hadronic decay modes of the di-tau system. The signal regions are split according to the Higgs production process into the categories 'VBF' (vector-boson fusion) and 'boosted' (gluon-gluon fusion). The splitting allows to adjust the event selection criteria such that the signal sensitivity can be optimized.

\subsection{Event selection and background estimation}

The preselection requires exactly two leptons $(=\tau, \mu, \mathrm{e})$ with opposite charge, missing transverse energy $E_{\mathrm{T}}^{\text {miss }}>20 \mathrm{GeV}$ (higher in the full-leptonic channel) and a veto on $b$-tagged jets (not in the full-hadronic channel). The VBF signal region requires at least two jets and a large invariant mass of the di-jet system. It is further split into subcategories with tighter and looser selection criteria. The tight VBF region selects events with a high di-jet mass, and a high transverse momentum $p_{\mathrm{T}}$ of the Higgs boson in the semi-leptonic channel. The boosted signal regions collect events with a large $p_{\mathrm{T}}$ of the Higgs boson and a high- $p_{\mathrm{T}}$ recoil jet which originates from the boost of the Higgs boson. The boosted signal regions are split further into a high- $p_{\mathrm{T}}^{\tau \tau}$ region with a higher Higgs $p_{\mathrm{T}}$ and a small angular distance between the taus and a low- $p_{\mathrm{T}}^{\tau \tau}$ region. In the full-hadronic mode, an additional VBF high- $p_{\mathrm{T}}^{\tau \tau}$ region is used because it provides a high signal purity. This categorization leads to 13 signal regions in total. Figure 1 gives an overview of the signal and background composition in the signal and control regions.

The Drell-Yan process $\left(Z / \gamma^{*} \rightarrow \tau \tau\right)$ represents the dominant irreducible background in all analysis categories with $50-90 \%$ of the total background. The second largest background in most signal regions originates from $W+$ jets and multijet processes. Further significant background contributions come from $Z \rightarrow \ell \ell$ and top events. In order to constrain the normalizations for the $Z \rightarrow \ell \ell$ and the top background from data, respective control regions are constructed. The $Z \rightarrow \ell \ell$ regions are defined by a di-lepton mass requirement of 


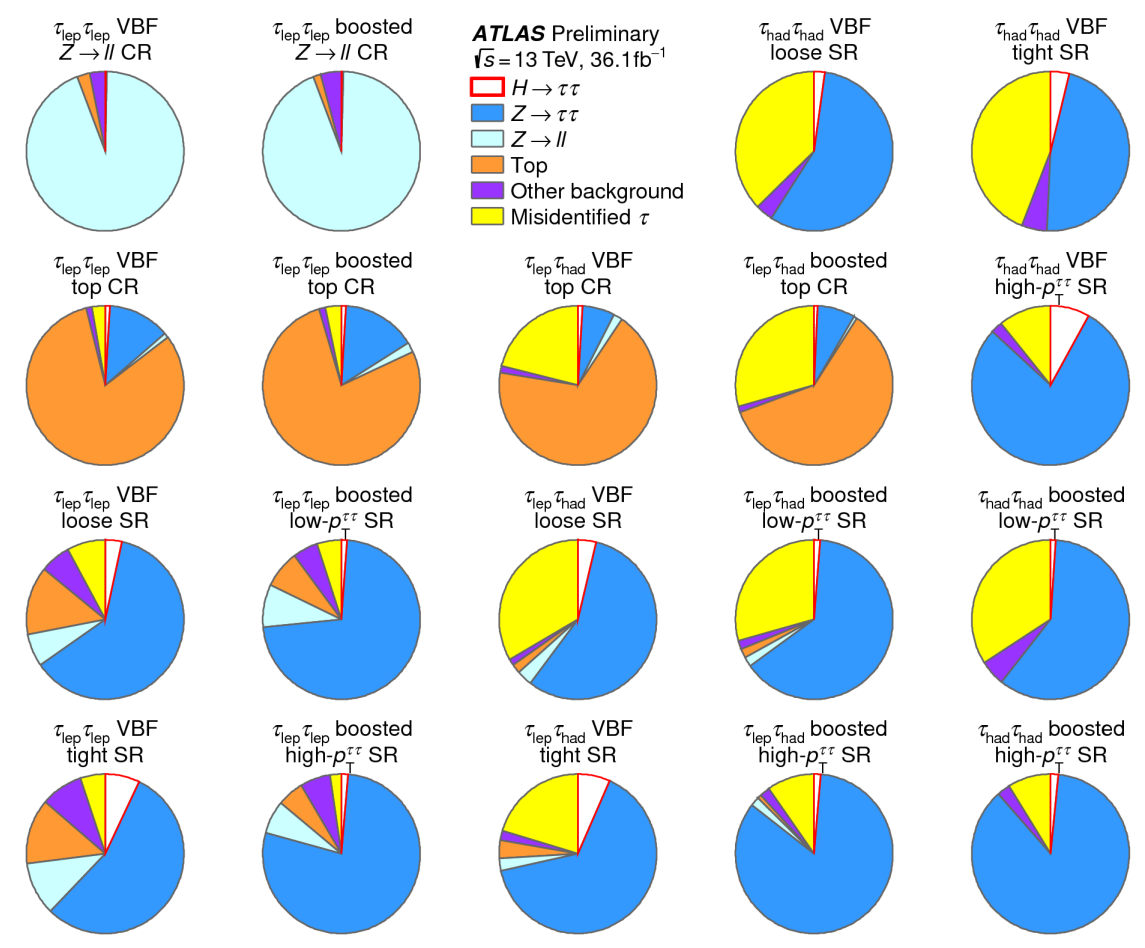

Figure 1: Expected signal and background composition in the 13 signal regions (SRs) and the six control regions (CRs). [7]

$80 \mathrm{GeV}<m_{\ell \ell}<100 \mathrm{GeV}$ and the top control regions are constructed by inverting the $b$-jet veto. For determining the normalizations, each control region is modelled by a single Poisson distribution to obtain the total expected event count in the global fit.

The $Z \rightarrow \tau \tau$, the $Z \rightarrow \ell \ell$ and the top background shapes are estimated from simulation, while a data-driven estimate is used for the background from misidentified taus. The datadriven background estimate is extracted from $W$-enriched regions. A fake-factor method is used in the semi-leptonic channel. In order to estimate the dominant background of real taus originating from the $Z \rightarrow \tau \tau$ decay, the $Z \rightarrow \tau \tau$ modelling is validated in regions with high purity in $Z \rightarrow \ell \ell$ events and similar kinematics to the $Z \rightarrow \tau \tau$ kinematics in the signal regions.

\subsection{Fit results and uncertainties}

The observable used in the fit is the di-tau mass. Due to the presence of neutrinos in the final state of the tau decay, advanced techniques for the di-tau mass reconstruction are required. For this purpose, the Missing Mass Calculator (MMC) [14] is used which provides a fine mass resolution of the di-tau resonance. It takes into account the mass and decay kinematics of the tau leptons and minimizes a likelihood function defined in the kinematically allowed phase space.

The signal strength, defined as the ratio of measured signal yield to the Standard Model expectation, is evaluated to be

$$
\left.\left.\mu_{H \rightarrow \tau \tau}=1.09_{-0.17}^{+0.18} \text { (stat.) }\right)_{-0.22}^{+0.27} \text { (syst.) }\right)_{-0.11}^{+0.16} \text { (theory syst.). }
$$

An excess of events with respect to the SM-background-only hypothesis is found by the likelihood fit with an observed (expected) significance of 4.4 (4.1) standard deviations. The result is compatible with a Standard Model Higgs boson with a mass of $125 \mathrm{GeV}$. Combined with the results from the previous measurement of the $H \rightarrow \tau \tau$ decay in ATLAS using data col- 
lected at $\sqrt{s}=7 \mathrm{TeV}$ and $8 \mathrm{TeV}$, the observed (expected) significance yields 6.4 (5.4) standard deviations.

The cross section defined as $\sigma_{H \rightarrow \tau \tau} \equiv \sigma_{H} \cdot \mathcal{B}(H \rightarrow \tau \tau)$ is extracted as parameter of interest from a maximum likelihood fit. The measured value is

$$
\left.\sigma_{H \rightarrow \tau \tau}=3.71_{-0.59}^{+0.60} \text { (stat.) }\right)_{-0.74}^{+0.87} \text { (syst.)pb. }
$$

A two-parameter cross-section fit separating the Higgs production processes is performed and yields $\sigma_{H \rightarrow \tau \tau}^{g g H}=3.0 \pm 1.0$ (stat.) $)_{-1.2}^{+1.6}$ (syst.)pb for the gluon-gluon-fusion process and $\sigma_{H \rightarrow \tau \tau}^{V B F}=0.28 \pm 0.09$ (stat.) ${ }_{-0.09}^{+0.11}$ (syst.)pb for the vector-boson-fusion process. Figure 2 shows the fit results in the three analysis channels and their combination in comparison with the Standard Model expectation, and the two-dimensional 68\% and 95\% confidence level (CL) contours in the plane of $\sigma_{H \rightarrow \tau \tau}^{V B F}$ versus $\sigma_{H \rightarrow \tau \tau}^{g g H}$.
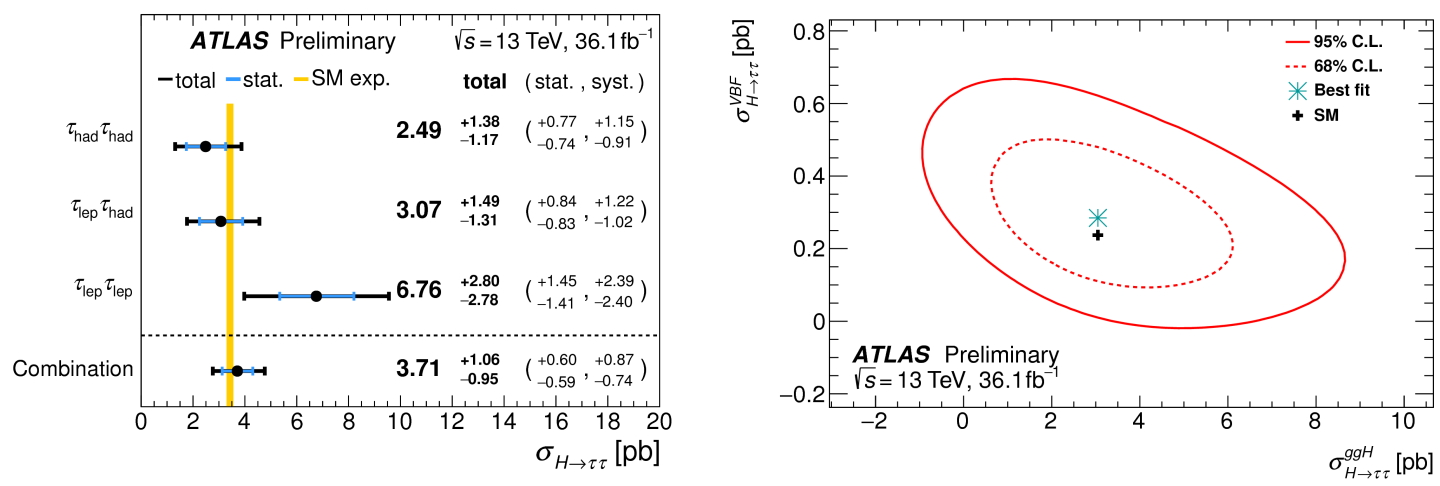

Figure 2: (a) Measured values for $\sigma_{H \rightarrow \tau \tau}$ from individual channels. Also shown is the result from the combined fit. The theory uncertainty in the predicted signal cross section is shown by the yellow band. (b) Likelihood contours for the combination of all channels in the depicted plane. [7]

\section{Search for the $H \rightarrow \mu \mu$ decay}

A search for the rare decay of the Higgs boson to dimuons in proton-proton collisions [11] has been performed at a centre-of-mass energy of $\sqrt{s}=13 \mathrm{TeV}$ with the ATLAS Detector. The collected data corresponds to an integrated luminosity of $79.8 \mathrm{fb}^{-1}$. The mass of the Higgs boson is assumed to be $m_{H}=125 \mathrm{GeV}$ for the presented results.

In this analysis, events are selected which have two isolated muons with opposite charges. The events are classified into ggF- and VBF-enriched regions, in total eight mutually exclusive categories. A Boosted Decision Tree (BDT) is used to maximize the separation between VBF signal and total background. It is used to select events for two VBF categories. All other events are categorized into six ggF categories based on the di-muon $p_{\mathrm{T}}$ and the muon pseudorapidities $\eta$. Figure 3(a) shows the BDT score used for the categorization, with the VBF signal peaking at 1 while the ggF signal and the background processes accumulate at low values.

The dominant background arises from the Drell-Yan process $Z / \gamma^{*} \rightarrow \mu \mu$. The Drell-Yan background prediction is reweighted to data according to the di-muon transverse momentum in order to improve the modelling. The minor background processes are fixed to the SM predictions. In this analysis, signal and background are modelled by analytic functions. In order to derive the functions, Monte-Carlo simulations are used. 

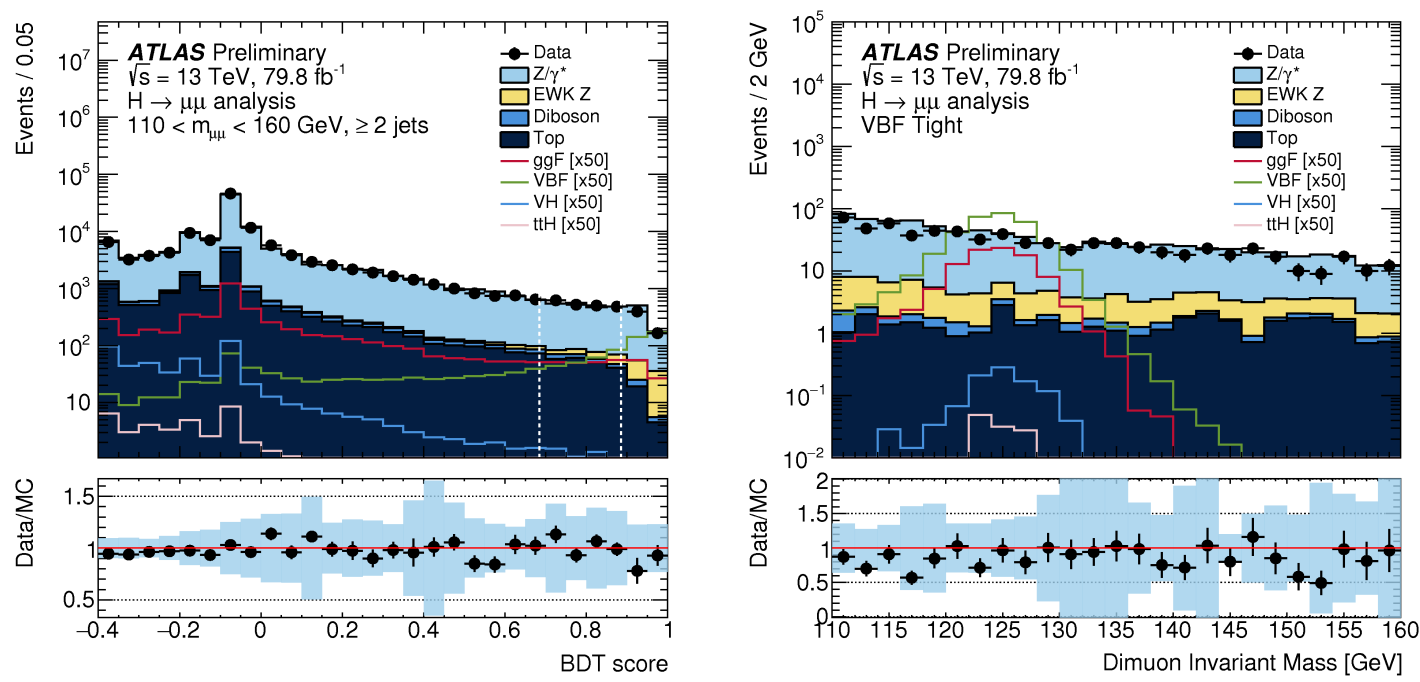

Figure 3: (a) Observed and simulated distributions of the BDT output score. The VBF tight and VBF loose categories are indicated by the vertical dashed lines. (b) Observed and simulated di-muon invariant mass spectra in the VBF tight signal region. [11]

The di-muon spectra in the eight categories are considered simultaneously in a fit of the signal-plus-background model to data. In order to avoid the $Z$ boson mass peak, the fit is performed in the mass window $110 \mathrm{GeV} \leq m_{\mu \mu} \leq 160 \mathrm{GeV}$ around the Higgs mass. Figure 3(b) shows the di-muon invariant mass spectra in the VBF tight signal region. The measured overall signal strength is $\mu=0.1_{-1.1}^{+1.0}$ and the observed (expected) significance for a Standard Model $H \rightarrow \mu \mu$ signal is 0.0 (0.9) standard deviations. The observed upper limit on the signal strength at 95\% CL is found to be 2.1 , while for the case of no $H \rightarrow \mu \mu$ signal, the expected upper limit is 2.0 and in the case of a Standard Model like $H \rightarrow \mu \mu$ signal the expected limit is 2.8 . The limit is dominated by the statistical uncertainty.

\section{Search for lepton-flavour-violating Higgs boson decays}

The analysis [13] presents the search for $H \rightarrow e \tau_{\text {had }}$ decays with one hadronically decaying tau lepton in the final state and a simultaneous search for the $H \rightarrow e \tau_{\text {lep }}$ and $H \rightarrow \mu \tau_{\text {lep }}$ decays with leptonically decaying tau leptons. The searches are performed on a dataset of protonproton collisions at a centre-of-mass energy of $\sqrt{s}=8 \mathrm{TeV}$. The data was collected with the ATLAS Detector and corresponds to an integrated luminosity of $20.3 \mathrm{fb}^{-1}$.

The techniques used for the searches in the $\tau_{\text {had }}$ analyses are similar to the techniques developed for the Standard Model $H \rightarrow \tau_{\text {lep }} \tau_{\text {had }}$ analysis presented in Section 2. In Figure 4, the post-fit $m_{\mathrm{MMC}}$ distribution is shown for the $e \tau_{\text {had }}$ channel.

In the search for lepton-flavour-violating decays of the Higgs boson, no significant excess was observed. Upper limits are set on the branching ratios for lepton-flavour-violating Higgs boson decays. The observed (median expected) $95 \%$ CL limits on $\mathcal{B}(H \rightarrow e \tau)$ are $1.04 \%$ $\left(1.21_{-0.34}^{+0.49} \%\right)$. In the search for $H \rightarrow \mu \tau$ decays, the observed (median expected) upper limits on $\mathcal{B}(H \rightarrow \mu \tau)$ are $1.43 \%\left(1.01_{-0.29}^{+0.40 \%)}\right.$. 


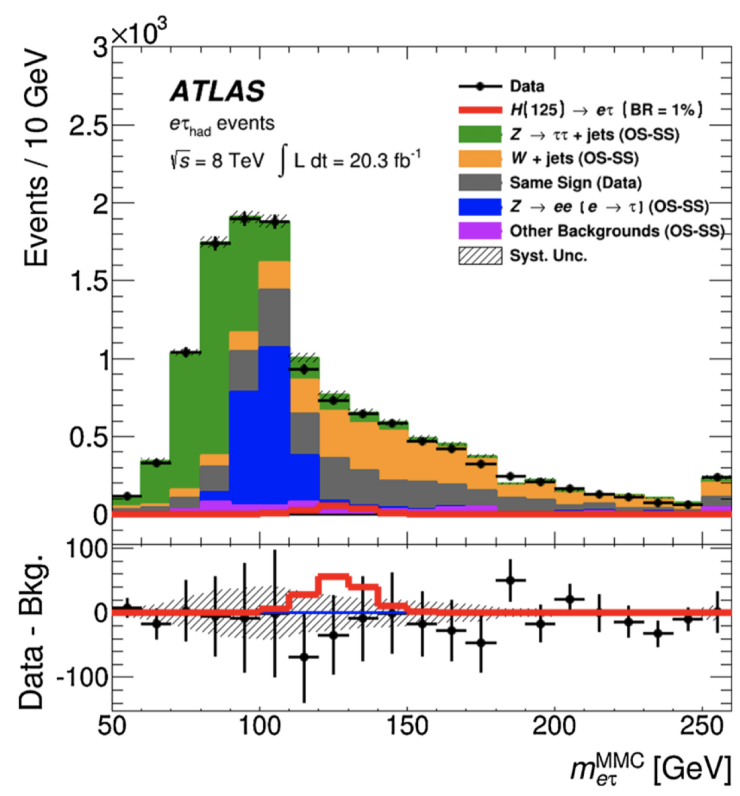

Figure 4: Post-fit combined $m_{\mathrm{MMC}}$ distribution in the $e \tau_{\text {had }}$ channel obtained by adding individual distributions in two signal regions. In the lower part of the figure, the data are shown after subtraction of the estimated backgrounds. The signal is shown assuming $\mathcal{B}(H \rightarrow e \tau)=1.0 \%$. [13]

\section{Conclusion}

In this article, three analyses have been presented with results from new measurements of decays of the Higgs boson to leptons. The data has been recorded with the ATLAS experiment at LHC.

In the measurement of the total Higgs boson production cross sections in proton-proton collisions at $\sqrt{s}=13 \mathrm{TeV}$ in the $H \rightarrow \tau \tau$ decay, an excess of events over the expected background from other Standard Model processes is found. It has an observed (expected) significance of 4.4 (4.1) $\sigma$. In combination with the results from the previous ATLAS measurement using data collected at $\sqrt{s}=7 \mathrm{TeV}$ and $8 \mathrm{TeV}$, the observed (expected) significance amounts to 6.4 (5.4) $\sigma$. This constitutes an observation of $H \rightarrow \tau \tau$ decays by the ATLAS experiment. The measured cross sections are in agreement with the Standard Model values.

The search for the rare decay of the Higgs boson to di-muons in proton-proton collisions has found no significant excess. More stringent upper limits could be determined for the $H \rightarrow \mu \mu$ signal strength.

No excess in data could be found for lepton-flavour-violating Higgs boson decays but stronger limits could be set on the lepton-flavour-violating Higgs boson decays which would represent new physics beyond the Standard Model.

Both, the results from the measurement of di-muon decays and those from the measurement of lepton-flavour-violating Higgs boson decays are in agreement with the Standard Model expectations.

Funding information Funded by BMBF-Forschungsschwerpunkt 103. 


\section{References}

[1] ATLAS Collaboration, The ATLAS experiment at the CERN Large Hadron Collider, J. Inst. 3, S08003 (2008), doi:10.1088/1748-0221/3/08/S08003.

[2] CMS Collaboration, The CMS experiment at the CERN LHC, J. Inst. 3, S08004 (2008), doi:10.1088/1748-0221/3/08/S08004.

[3] ATLAS Collaboration, Observation of a new particle in the search for the Standard Model Higgs boson with the ATLAS detector at the LHC, Phys. Lett. B 716, 1 (2012), doi:10.1016/j.physletb.2012.08.020.

[4] CMS Collaboration, Observation of a new boson at a mass of $125 \mathrm{GeV}$ with the CMS experiment at the LHC, Phys. Lett. B 716, 30 (2012), doi:10.1016/j.physletb.2012.08.021.

[5] ATLAS Collaboration, Evidence for the Higgs-boson Yukawa coupling to tau leptons with the ATLAS detector, J. High Energ. Phys. 04, 117 (2015), doi:10.1007/JHEP04(2015)117.

[6] CMS Collaboration, Evidence for the $125 \mathrm{GeV}$ Higgs boson decaying to a pair of $\tau$ leptons, J. High Energ. Phys. 05, 104 (2014), doi:10.1007/JHEP05(2014)104.

[7] ATLAS Collaboration, Cross-section measurements of the Higgs boson decaying to a pair of tau leptons in proton-proton collisions at $\sqrt{s}=13 \mathrm{TeV}$ with the ATLAS detector, Tech. Rep. ATLAS-CONF-2018-021, CERN, Geneva (2018), https://cds.cern.ch/record/2621794.

[8] CMS Collaboration, Observation of the Higgs boson decay to a pair of $\tau$ leptons with the CMS detector, Phys. Lett. B 779, 283 (2018), doi:10.1016/j.physletb.2018.02.004.

[9] ATLAS Collaboration, Search for the dimuon decay of the Higgs boson in pp collisions at $\sqrt{s}=13 \mathrm{TeV}$ with the ATLAS detector, Phys. Rev. Lett. 119, 051802 (2017), doi:10.1103/PhysRevLett.119.051802.

[10] CMS Collaboration, Search for the Higgs boson decaying to two muons in proton-proton collisions at $\sqrt{s}=13 \mathrm{TeV}$, Tech. Rep. CMS-HIG-17-019-003, CERN, Geneva (2018), https: //cds.cern.ch/record/2631546.

[11] ATLAS Collaboration, A search for the rare decay of the Standard Model Higgs boson to dimuons in pp collisions at $\sqrt{s}=13 \mathrm{TeV}$ with the ATLAS Detector, Tech. Rep. ATLASCONF-2018-026, CERN, Geneva (2018), https://cds.cern.ch/record/2628763.

[12] ATLAS Collaboration, Search for lepton-flavour-violating $H \rightarrow \mu \tau$ decays of the Higgs boson with the ATLAS detector, J. High Energ. Phys. 11, 211 (2015), doi:10.1007/JHEP11(2015)211.

[13] ATLAS Collaboration, Search for lepton-flavour-violating decays of the Higgs and $Z$ bosons with the ATLAS detector, Eur. Phys. J. C 77, 70 (2017), doi:10.1140/epjc/s10052-0174624-0.

[14] A. Elagin, P. Murat, A. Pranko and A. Safonov, A new mass reconstruction technique for resonances decaying to $\tau \tau$, Nucl. Instrum. Methods Phys. Res. Sect. A 654, 481 (2011), doi:10.1016/j.nima.2011.07.009. 